Revue de l'Institut des langues et cultures

d'Europe, Amérique, Afrique, Asie et Australie

44 | 2021

Peinture, identité nationale et style international en

Europe autour de 1900

\title{
L'internationalisation des expositions parisiennes et la crise du système du Salon à la fin du XIX ${ }^{e}$ siècle
}

The Internationalization of Parisian Exhibitions and the Crisis of the Salon System at the end of the 19th Century

Laurent Cazes

\section{OpenEdition}

Journals

Édition électronique

URL : https://journals.openedition.org/ilcea/12449

DOI : $10.4000 /$ ilcea.12449

ISSN : 2101-0609

Éditeur

UGA Éditions/Université Grenoble Alpes

Édition imprimée

ISBN : 978-2-37747-324-3

ISSN : 1639-6073

Référence électronique

Laurent Cazes, «L'internationalisation des expositions parisiennes et la crise du système du Salon à la fin du xixe siècle », ILCEA [En ligne], 44 | 2021, mis en ligne le 02 novembre 2021, consulté le 02 décembre 2021. URL : http://journals.openedition.org/ilcea/12449 ; DOI : https://doi.org/10.4000/ ilcea. 12449

Ce document a été généré automatiquement le 2 décembre 2021

(C) ILCEA 


\title{
L'internationalisation des
} expositions parisiennes et la crise du système du Salon à la fin du XIX ${ }^{\mathrm{e}}$ siècle

\author{
The Internationalization of Parisian Exhibitions and the Crisis of the Salon \\ System at the end of the 19th Century
}

\section{Laurent Cazes}

\section{Introduction}

1 Depuis une quarantaine d'années, les nombreux travaux consacrés au Salon et plus largement à la vie artistique du xix ${ }^{e}$ siècle ont permis d'enrichir la connaissance du rôle majeur de cette institution pour le développement, la diffusion et la promotion de l'art contemporain. Le cliché d'une institution désuète qui se serait contenté d'entraver le développement de l'art moderne appartient désormais au passé ${ }^{1}$. D'autre part l'importance des phénomènes d'échanges et d'internationalisation artistiques apportés par les expositions internationales de la seconde moitié du XIX siècle a été très tôt prise en compte et continue de faire l'objet de nouvelles recherches (Brunet \& Talley, 2017 ; Boussahba-Bravard \& Rogers, 2018).

2 L'historiographie du Salon parisien reste pourtant encore en grande partie cantonnée à la sphère française, alors que l'exposition connut également un rayonnement international de premier plan. Les restes d'un a priori sur l'hermétisme de l'institution, associé à un raccourci fréquent mais trompeur entre "nationalisme» et " académisme ${ }^{2}$ ", peut expliquer - avec le fait que l'historiographie traditionnelle privilégie l'étude de figures considérées comme indépendantes ou d'« avant-garde " (Joyeux-Prunel, 2016) - que le Salon soit resté relativement en marge des études transnationales. 
3 Le rôle majeur de l'exposition au Salon pour la carrière des artistes étrangers apparaît ainsi dans de nombreux travaux, en particulier concernant les peintres américains (Fink, 1990), italiens (Lagrange, 2010) et espagnols (Karp-Lugo, 2014). Les artistes étrangers étaient désirés au Salon parisien, où ils exposèrent en nombre ${ }^{3}$, en particulier au cours de la seconde moitié du xix ${ }^{e}$ siècle, et eurent une influence certaine sur le développement de l'art français, notamment dans le domaine de la peinture de genre (Mainardi, 1987 ; Vottero, 2012).

4 À travers le prisme de la participation des peintres étrangers, cet article propose d'évoquer comment le Salon, associé aux expositions universelles françaises à partir de 1855 , constitua un véritable système de promotion des carrières artistiques à l'échelle internationale. Ce système établit une élite artistique cosmopolite qui domina les grandes expositions de la seconde moitié du XIX ${ }^{e}$ siècle, mais montra ses limites à partir des années 1880. Le désengagement de l'État de l'organisation du Salon entraîna un délitement de cette élite artistique et sa dispersion entre le Salon traditionnel et celui de la Société nationale des beaux-arts, créé en 1889. Tandis que le régime républicain amena à clarifier le statut de l'exposant étranger dans la sphère artistique parisienne, l'art cosmopolite du Salon qui brilla intensément durant les premiers Salons de la Société nationale des beaux-arts se trouva bientôt délaissé par les artistes et la critique. Le système du Salon laissa place à la fin du xix siècle à une nouvelle carte des expositions qui se caractérise par sa diversité et une autonomie croissante.

\section{Aux origines d'une scène artistique internationale à Paris : Salons et expositions universelles}

5 Depuis la fin de l'Ancien Régime, le Salon avait officiellement ouvert ses portes aux artistes étrangers, mais ce ne fut qu'à partir du Second Empire que ces derniers y exposèrent en nombre et furent appréhendés comme une véritable composante de l'exposition ${ }^{4}$. Ainsi à partir de 1852, les livrets du Salon mentionnent de façon systématique le lieu de naissance et le lieu de formation ou le nom du maitre des exposants. Chaque artiste de l'exposition pouvait être désormais rattaché à un pays, une école nationale ou à un maître ${ }^{5}$.

6 À l'origine de cette initiative se trouvait le comte de Nieuwerkerke, nommé à la tête de l'administration des beaux-arts de Napoléon III, et surtout Philippe de Chennevières, nouvellement en charge de l'organisation du Salon. Tout au long de sa carrière, ce dernier accorda une attention particulière à l'art étranger exposé au Salon et à l'acquisition de ces œuvres pour l'enrichissement des collections françaises. Cette attention ressort notamment d'une lettre qu'il adressa au comte de Nieuwerkerke à la suite du Salon de 1853, pour lui signaler l'importance qu'avaient prise cette année-là les œuvres des artistes étrangers, en particulier les artistes belges. Chennevières évoque une " concurrence " pour les artistes français, mais insiste sur le caractère positif que celle-ci constitue à ses yeux : la présence étrangère apporte du prestige à l'exposition ; la participation des artistes belges peut entraîner à sa suite celles d'artistes d'autres nationalités; enfin cette présence permettra, par un phénomène d'émulation, d'élever la qualité de la production française :

Il me semble donc, Monsieur le Directeur général, que nous avons à nous féliciter, sous un triple rapport, de l'éclatant succès des artistes Belges au Salon de 1853 :

$1^{\circ}$ parce qu'ils donnent à votre Exposition une grande part de son lustre ; $2^{\circ}$ parce 
qu'ils montrent le chemin des Expositions de Paris aux meilleurs artistes du reste de l'Europe, et, enfin, parce qu'ils vont éveiller dans nos artistes Français un utile sentiment de rivalité et une salutaire préoccupation de notre supériorité nationale (Chennevières : 1853).

7 Cette politique d'internationalisation des expositions officielles prit quelques années plus tard une dimension sans précédent, avec l'organisation de l'Exposition universelle de 1855. Conçue à la suite de l'exposition britannique de 1851, cette manifestation devait au départ être consacrée uniquement aux produits de l'industrie ; cependant, afin de se démarquer du précédent anglais, le gouvernement français décida d'y intégrer les beaux-arts, domaine dans lequel la France se sentait mieux à même de faire valoir ce qu'elle considérait comme une suprématie. Aussi le gouvernement décréta-t-il l'annulation du Salon de 1854 et son remplacement l'année suivante par l'Exposition universelle ${ }^{6}$.

8 Assimiler le Salon à l'Exposition universelle de 1855 eut un impact considérable dans l'affirmation de Paris comme capitale artistique. Le Salon renforça le prestige de l'Exposition universelle, et celle-ci renforça en retour le prestige du Salon. Jamais un aussi grand nombre d'œuvres étrangères contemporaines n'avait fait le voyage jusqu'à Paris; le nombre de visiteurs, français et surtout étrangers, était également sans précédent.

9 Après l'événement de 1855 et la découverte de la richesse de la production étrangère qui y avait été exposée dans les différents pavillons nationaux, le Salon connut une nouvelle dimension. L'exposition bénéficiait d'un nouveau lieu, le Palais de l'industrie construit pour l'Exposition de 1855, et se tint désormais selon un rythme bisannuel, avant de retrouver une fréquence annuelle à partir 1863. La critique était aussi plus attentive à la production étrangère qui y était exposée, la dimension internationale devenant un véritable critère d'appréciation de l'exposition dans son ensemble.

Les expositions universelles suivantes, celles de 1867, 1878, 1889 et 1900 se tinrent séparément du Salon, et il est nécessaire de bien distinguer les deux types d'expositions. L'exposition au Salon relevait d'une démarche individuelle de l'artiste, qui soumettait ses œuvres au jury, tandis qu'un comité propre à chaque pays sélectionnait les exposants des différentes sections nationales aux expositions universelles. Et contrairement à cet accrochage déterminé par la nationalité des artistes, l'accrochage au Salon mêlait sans distinction artistes français et artistes étrangers ${ }^{7}$.

11 Cependant l'analyse des listes d'exposants et du cheminement de leurs carrières permet de faire ressortir un lien étroit entre Salons et expositions universelles. Certains peintres qui exposaient à Paris pour la première fois en 1855, exposèrent par la suite très régulièrement Salon. Ce fut par exemple le cas du Néerlandais Jozef Israëls ou de l'Allemand August Friedrich Schenck, célèbre pour ses peintures animalières, qui s'établit en France au début des années 1860. Mais il apparaît surtout que les peintres étrangers sélectionnés pour les expositions universelles suivantes s'étaient fait connaître en exposant auparavant au Salon. Ainsi lors de l'Exposition universelle de 1867, Lawrence Alma-Tadema, (qui n'était pas encore naturalisé anglais) et Jozef Israëls exposaient dans la section des Pays-Bas; Oswald Achenbach et Ludwig Knaus dans la section de la Prusse et de l'Allemagne du Nord; Albert Anker dans la section de la Suisse ; Giuseppe Palizzi et Alberto Pasini dans la section italienne... Or tous ces artistes s'étaient fait connaître au Salon, où ils exposaient avec succès, parfois depuis plus d'une 
dizaine d'années ${ }^{8}$. Aussi de nombreux critiques firent-ils part de leur frustration, face au peu de nouveauté que présentaient à leurs yeux les sections étrangères de 1867, comparativement à la manifestation de 1855 .

On constate ainsi, jusque dans les années 1890 , une homogénéisation des exposants étrangers aux Salons et aux exposition universelles parisiennes. Cela indique l'impact de l'exposition au Salon pour la reconnaissance des artistes étrangers, y compris dans leur pays d'origine. Tandis qu'en 1855, des renommées étrangères établies au sein des académies de leur pays d'origine avaient été exposées à Paris; en 1867, un certain nombre de renommées étrangères s'étant établies au Salon parisien, avaient de ce fait intégré la section nationale de leur pays d'origine. Le même phénomène se vérifie pour l'Exposition universelle de 1878, où l'on retrouve notamment Frederick Arthur Bridgman, présent au Salon depuis 1868, dans la section des États-Unis; Giuseppe de Nittis, présent au Salon depuis 1870, dans la section italienne; ou encore Miháli Munkácsy dans la section hongroise. Ce dernier avait connu en 1870 un succès fulgurant avec son premier envoi au Salon, intitulé Le Dernier jour d'un condamné (Budapest, musée national hongrois).

13 Salons et expositions universelles à Paris firent ainsi naître une élite artistique qui dominait la scène internationale. À partir de 1864, cette élite apparaît de façon exhaustive dans une «listes par ordre alphabétique des artistes récompensés vivants » placée au début de chaque livret de Salon, mentionnant pour chacun d'entre eux les médailles et décorations obtenues aux deux types d'expositions9.

Les récompenses constituaient en effet la clé de voûte de ce système de promotion artistique. Sans entrer dans les détails des aléas du règlement, qui furent nombreux au cours de la période, il existait une équivalence entre les médailles d'or, d'argent et de bronze délivrées à l'issue des expositions universelles et les trois classes de médailles délivrées à l'issue du Salon. Loin d'être anecdotiques, médailles et décorations étaient cruciales en ce qu'elles constituaient le véritable critère de reconnaissance pour un artiste; aussi leur hiérarchie était-elle prise très au sérieux ${ }^{10}$. De plus, médailles et décorations (là aussi de façon variable selon les aléas du règlement) permettaient à un artiste d'être exempté de jury, c'est-à-dire d'être admis d'office au Salon.

En agissant comme un véritable système de promotion des carrières, Salons et expositions universelles firent de Paris une scène artistique à l'attraction et au dynamisme croissants pour les artistes du monde entier. Ce système prouva son efficacité de façon durable, le Salon donnant le rythme et l'orientation de la création artistique contemporaine, en France comme à l'étranger, tout au long de la seconde moitié du $\mathrm{XIX}^{\mathrm{e}}$ siècle. Cependant face au nombre grandissant des artistes exposants; face à la diversification des styles et des orientations esthétiques, et dans un contexte global de montée des nationalismes, ce système allait aussi montrer ses limites au cours des deux dernières décennies.

\section{Le tournant républicain et la crise du système}

16 Les événements de 1870 n'apportèrent pas de modification majeure au Salon lorsqu'il rouvrit en 1872. Si l'on observe une diminution de la participation étrangère dans les premières années, celle-ci retrouve dès 1875 la même proportion que sous le Second Empire par rapport au reste des exposants. Sa composition n'était cependant plus la même, avec une très faible participation des peintres allemands, et l'émergence 
notamment du groupe des peintres scandinaves, qui connurent un grand succès au début de la Troisième République; et du groupe des peintres américains, qui composaient l'ensemble le plus important des exposants étrangers dans les Salons de la fin du siècle (graph. 2).

Ce n'est qu'avec la fin de la période de l'Ordre moral et l'arrivée des républicains au pouvoir qu'une réforme du Salon fut mise en œuvre par Edmond Turquet, nommé à la direction du sous-secrétariat d'État aux beaux-arts. Ces réformes, mises en place pour le Salon de 1880, avaient pour but de « républicaniser » le Salon : élargissement du droit de vote pour l'élection du jury; valorisation de l'art monumental, considéré comme négligé sous les régimes précédents... souhaitant également favoriser la dimension éducative de l'exposition, Turquet décida d'abolir le système d'accrochage par ordre alphabétique d'auteur, qui prévalait depuis 1861 , au profit d'un classement qui se voulait didactique. Ainsi les œuvres furent exposées séparées en quatre catégories, selon le statut de l'auteur: artistes hors concours; artistes exemptés de jury d'admission; artistes étrangers; la dernière catégorie rassemblant le reste des exposants.

Ces réformes furent très mal accueillies et les dissensions avec la nouvelle administration des beaux-arts devinrent si fortes qu'Edmond Turquet pris la décision radicale de remettre désormais la gestion du Salon à l'entière responsabilité des artistes. À partir de 1881, l'exposition fut ainsi gérée par la nouvelle «Société des artistes français ».

19 L'historiographie a apporté diverses interprétations de l'impact qu'eut le désengagement de l'État de l'organisation du Salon. Patricia Mainardi a souligné l'importance symbolique de cette date, affirmant qu'elle marquait un « effondrement du système ", c'est à dire la fin des expositions officielles en France, et avec elle, celle des honneurs et des médailles. Autrement dit le désengagement de l'État aurait entraîné la disparition de cette élite d'artistes privilégiés qu'avaient fait émerger Salons et exposition universelles depuis 1855 (Mainardi, 1993). Dans une analyse plus nuancée, Pierre Vaisse a montré que l'impact de cette décision n'eut que peu d'effet dans un premier temps. De fait, le Salon continua de fonctionner de la même façon qu'auparavant, dans le même lieu, avec un règlement et un jury similaires, et les mêmes exposants. De plus, la Société des artistes français fit son possible pour conserver de façon symbolique le patronage de l'État, le gouvernement continuant d'effectuer des achats officiels au Salon, et assistant toujours aux vernissages et aux cérémonies de remise des récompenses. L'impact de la décision de Turquet n'apparut réellement qu'une dizaine d'année plus tard, au moment de l'Exposition universelle de 1889 (Vaisse, 2010).

Le désengagement de l'État de l'organisation du Salon avait en effet rendu caduc le lien traditionnel entre Salons et expositions universelles. Aussi les membres de la Société des artistes français ne furent-ils que partiellement intégrés au jury d'admission et des récompenses de l'Exposition universelle de 1889. L'administration des beaux-arts avait nommé à la tête du jury des récompenses Ernest Meissonier - membre de la Société des artistes français, mais avec laquelle il était en conflit, en particulier avec son président William Bouguereau, qui avait la réputation de régner en despote sur le Salon, au point que certains commentateurs appelaient désormais l'exposition le «Salon Bouguereau » (Lobstein, 2000). 
21 À l'issue de l'Exposition universelle de 1889, un violent conflit éclat au sein des membres de la Société des artistes français à propos des médailles décernées par le jury présidé par Meissonier. D'une part, la hiérarchie établie par les précédentes expositions n'avait pas été respectée : certains artistes avaient été "dégradés » en obtenant une médaille inférieure à celle(s) déjà obtenue(s); il y eut ainsi plusieurs lettres de refus venant d'artistes qui se considéraient humiliés. D'autre part, un nombre considérable de médailles avait été décerné, de sorte qu'il était difficile pour la Société des artistes français de les reconnaître dans le cadre du Salon annuel. En effet, si la règle traditionnelle d'équivalence des récompenses avait été appliquée à l'ensemble des artistes médaillés à l'issue de l'Exposition universelle de 1889, près de cinq cents artistes se seraient ajoutés à la liste déjà très longue des exemptés de jury d'admission.

22 Ainsi, d'un côté, les dirigeants de la Société des artistes français, Bouguereau en tête, s'opposaient à inscrire au nombre des exemptés les artistes médaillés de l'Exposition universelle de 1889 ; et de l'autre, des membres de la Société qui avaient, pour certains, participé au jury des récompenses de l'Exposition universelle, Meissonier en tête, souhaitaient les y inscrire.

23 Le conflit aboutit à la démission de Meissonier et d'une partie des membres de la Société des artistes français, qui fondèrent rapidement les statuts d'une nouvelle "Société nationale des Beaux-Arts", dont le premier Salon eut lieu dès 1890, dans le Palais des beaux-arts, récemment bâtit sur le Champ-de-Mars pour l'Exposition universelle ${ }^{11}$. L'unité artistique qu'avait jusque-là préservé le système du Salon se trouvait désormais dispersée dans deux expositions distinctes.

\section{Évolutions du statut de l'exposant étranger}

24 Faut-il attribuer cette dispersion de la scène artistique parisienne à son internationalisation croissante? Le conflit entre "meissonieristes» et "bouguereauistes ", comme l'appelaient les contemporains, ressemblerait plutôt à une querelle de chapelle franco-française. Le Salon était avant tout confronté au problème du nombre des exposants - indépendamment de leur nationalité - qui ne cessait de grandir depuis le milieu du siècle. Cependant la scission du Salon se déroula dans un contexte où le critère de la nationalité de l'artiste était en train d'évoluer, et la question de la participation étrangère en a été l'un des aspects les plus discutés.

Déjà au moment des réformes du Salon de 1880, le fait de séparer œuvres françaises et œuvres étrangères avait provoqué des plaintes de la part des exposants étrangers. De nombreux critiques leur avaient donné raison ; Émile Zola par exemple y vit une forme d'ostracisation :

Voici maintenant le groupe des étrangers qui se lamente. Ces artistes prétendent que, lorsqu'ils viennent exposer en France, c'est pour se trouver en compagnie des artistes français, et non pour être relégués à part, comme cela se pratique dans les expositions universelles. Je dirais que cette plainte me paraît assez juste. Beaucoup d'artistes étrangers entendent avant tout se mesurer avec les artistes français et regardent comme un honneur de marcher dans leurs rangs. Si on les met à part, c'est comme si on les laissait chez eux (Zola, 1970 [1880]).

Lorsqu'à la suite de l'échec de la réforme de 1880, l'organisation du Salon fut remise aux mains de la Société des artistes français, celle-ci adopta pour l'exposition de 1881 un retour à l'accrochage alphabétique et à un règlement qui ressemblait beaucoup à 
celui précédant les réformes de Turquet. On relève cependant parmi les quelques nouveautés un point qui semble a priori anecdotique, mais se révèle très significatif de l'évolution du statut de l'étranger en France au cours des années 1880. L'article cinq du nouveau règlement mentionne en effet le fait que les artistes, au moment du dépôt de leurs œuvres, doivent désormais indiquer leur «nationalité » en plus de leur lieu de naissance.

C'était la première fois, dans le cadre du règlement du Salon, que la distinction était faite entre le lieu de naissance d'un artiste et sa nationalité. Cela correspondait à une évolution globale de la législation française, qui allait aboutir à la loi sur la «nationalité française » de 1889, celle-ci comblant un vide concernant le statut des étrangers.

La catégorie des artistes étrangers au Salon, qui avait toujours été un peu floue, notamment en raison du fait que nombre d'entre eux vivaient en France, devint ainsi de mieux en mieux identifiée. Plusieurs revues de Salon de la période publièrent des statistiques documentant non seulement la part des exposants étrangers, mais aussi celle des différentes nationalités la composant. À partir de 1878, L'Annuaire statistique de la France publia également des chiffres sur le Salon, indiquant, entre autres, le nombre d'exposants étrangers, puis, à partir de 1882, le détail de leur nombre par catégories nationales (graph. 1 et 2).

Gérard Noiriel a montré que l'unité nationale engagée par la Troisième République posa la question du statut de l'étranger sous un jour nouveau (Noiriel, 1999). La prise en compte de l'ensemble des milieux sociaux, l'intérêt porté aux classes populaires par le régime républicain, attribuait à l'État la nouvelle fonction d'assurer et de protéger le droit social de tous les citoyens. Dès lors, la distinction s'imposait entre les citoyens " nationaux », qui avaient le droit de jouir de cette protection sociale, et les autres.

À partir des années 1880, le monde artistique parisien fut agité par des affaires illustrant le passage d'un libéralisme globalement ouvert aux étrangers, vers des réactions protectionnistes parfois violentes. Une brochure publiée en 1886 se fit notamment l'écho, extraits de lettres de plaintes d'étudiants et articles de presse à l'appui, d'une polémique à propos de l'Envahissement de l'École des Beaux-Arts par les Étrangers. Des élèves architectes de l'École accusaient certains de leurs camarades étrangers de conserver à dessein leur nationalité afin de s'affranchir des obligations militaires, pour ensuite se faire naturaliser français, gagnant ainsi plusieurs années pour réaliser leur cursus. La presse évoquait aussi une « invasion » des étrangers dans les ateliers de peintures, et même l'idée d'élèves français "expulsés par leurs hôtes " (Vérax, 1886).

31 Quelques années auparavant, en juillet 1883, une autre polémique avait embrasé la chronique artistique, quand le gouvernement américain décida de multiplier par trois la taxe d'importation des œuvres d'art, afin de soutenir la production artistique locale. La Société des artistes français, notamment, réagit vivement, et menaça dans un communiqué de prendre de nouvelles mesures concernant l'ouverture du Salon aux artistes étrangers - sans pour autant les mettre œuvre. 


\section{La Société nationale des beaux-arts, brève héritière du système du Salon?}

32 C'est dans ce contexte que se produisit la scission du Salon traditionnel et la création de la Société nationale des beaux-arts. Or, parmi les centaines d'artistes médaillés de 1889 que Meissonnier et ses partisans souhaitaient inscrire à la liste des exemptés du Salon des artistes français, se trouvaient essentiellement des artistes étrangers. L'ancien Salon donna ainsi l'impression de vouloir fermer ses portes aux étrangers. Aussi l'idée d'un chauvinisme de la Société des artistes français s'est-t-elle développée jusque dans l'historiographie récente (Maingon, 2009). Quelques-uns de ses membres firent certes preuve de chauvinisme. Par exemple, lors d'un débat du comité au moment de la crise, Ferdinand Humbert et Auguste Bartholdi avaient suggéré de ne refuser l'exemption qu'aux étrangers ; mais la proposition fut rejetée de façon catégorique (Tolède, 2008 : 35).

La scission de 1889 ne modifia pas les critères d'admission des exposants étrangers aux Salons des artistes français. Tout au long des années 1890, leur participation continua de représenter vingt pour cent environ des exposants (graph. 1). Cependant la scission de 1889 priva le Salon des artistes français de ses exposants étrangers les plus prestigieux, car les médaillés de 1889, reconnaissants envers le jury présidé par Meissonier, rejoignirent l'exposition de la Société nationale des beaux-arts.

L'Italien Boldini, l'Américain John Singer Sargent, le Finlandais Albert Edelfelt, le Belge Alfred Stevens, entre autres grandes renommées internationales, exposèrent en 1890 à la Nationale des beaux-arts, assurant d'emblée à ce nouveau Salon un prestige incontesté. Beaucoup de commentateurs, à l'instar de la Gazette des Beaux-Arts, évoquèrent une perte considérable pour le Salon des artistes français :

Presque forcément les artistes venus du dehors devaient suivre la troupe de Meissonier. Or, c'est là pour le salon des Champs-Élysées, une perte déplorable, irréparable. Point n'est besoin d'être très perspicace pour constater l'envahissement progressif, depuis dix ans, et le triomphe de plus en plus éclatant des artistes étrangers. Les noms de Edelfelt, de MM. de Uhde, Kroyer, Werenskiold, Israëls, Harrison, Pearce, Melchers, etc., etc., sont dans toutes les mémoires [...]. Eh bien, aucun de ces peintres n'expose au Salon, cette année; et de tous les trous béants, ceux-là, à notre avis, si la paix ne se signe pas, resteront les plus difficiles à boucher (Albert, 1890).

Les deux salons présentaient des physionomies très différentes. Celui de la Nationale était beaucoup plus réduit en nombre d'œuvres et d'artistes; le cadre y était plus luxueux, l'accrochage plus aéré. Mais le principal caractère du nouveau Salon était le brillant de son cosmopolitisme, tant par la renommée de ses exposants étrangers que par leur nombre, celui-ci représentant près de quarante pour cent de l'exposition, avec une plus grande diversité de nationalités (graph. 1 et 2).

La création de la Société nationale des beaux-arts a souvent été comparée à celles des Sécessions européennes de la fin du siècle (Tolède, 2008; Joyeux-Prunel, 2016), notamment en raison d'un système de sélection élitiste des exposants, associant à un jury d'admission des membres fondateurs, sociétaires et associés admis d'office. Mais d'un point de vue esthétique, le Salon de la Nationale n'était pas en rupture avec les expositions traditionnelles. Les artistes qui y exposaient étaient les mêmes que ceux de l'ancien Salon et des expositions universelles. De plus, Meissonier n'avait pas conçu la 
Société nationale des beaux-arts comme une société dissidente. Au contraire, il l'avait conçue dans un esprit de continuité avec l'Exposition universelle de 1889, et revendiquait pour cette raison le lien symbolique avec les expositions d'État, dont jouissait encore l'ancien Salon. Son objectif était simplement de remplacer le Salon des Artistes français, c'est pourquoi il avait donné à sa société le nom de «nationale des beaux-arts ${ }^{12} »$.

La scission de 1889 eut donc pour résultat de scinder l'élite artistique internationale des expositions parisiennes, Meissonier étant parvenu à en capter la majeure partie à travers les médaillés de l'Exposition universelle. Le succès fut immédiat, mais le salon de la Société nationale des beaux-arts montra rapidement les limites d'un cosmopolitisme artistique, dominé par le portrait mondain et les scènes naturalistes, qui n'était plus tout à fait en phase avec l'époque.

Dans une critique du Salon de la Nationale de 1892, Gustave Geffroy reproche aux œuvres étrangères de ne plus rien exprimer d'exotique. Citant les peintres scandinaves Anders Zorn, Albert Edelfelt et Peter Severin Krøyer, il dénonce une uniformisation des " procédés » et la parisianisation d'un art qui lui semble être l'œuvre d'habitants d'un même " pays du chic ». Cette peinture étrangère était à ses yeux d'autant plus suspecte que leurs célèbres auteurs s'étaient installés en nombre dans les nouveaux quartiers chics de la capitale :

Les étrangers, les voici : MM. Edelfelt, Kroyer et Zorn, et l'on serait fort empêché de dire par quoi ils se différencient de leurs collègues français. [...] Ils sont indifféremment de l'Allemagne ou de la Norwège [sic], de l'Angleterre ou de l'Espagne, de la Suisse ou de l'Amérique, et c'est extraordinaire de les voir cherchant à se naturaliser habitants du même pays du chic, de la blague, de la besogne vite faite, sans attention, sans sincérité. Ils habitent tous pêle-mêle la même avenue de Villiers artistique et s'il y a une remarque à faire, c'est que les étrangers suivent peut-être les modes régnantes avec plus de précipitation que les indigènes (Geffroy, 1893).

Parallèlement à cette évolution du goût, apparaissaient les Sécessions européennes : Munich en 1892, Vienne en 1897, Berlin en 1898, où se développèrent des scènes artistiques très actives. Or on constate que de nombreux artistes phares cessèrent d'exposer au Salon de la Nationale des beaux-arts à partir du milieu des années 1890 . Ce fut par exemple le cas des Allemands Max Liebermann et Fritz von Uhde, qui privilégiaient désormais une activité dans leur pays d'origine.

De ce point de vue, le Salon de la Société nationale des beaux-arts apparaît moins comme une « sécession » à la française que comme le chant du cygne d'un «système du Salon" qui s'éteignit avec le $\mathrm{xIX}^{\mathrm{e}}$ siècle. Et la dispersion de l'élite artistique des exposition parisiennes fut suivie d'une multiplication des foyers artistiques, dessinant une nouvelle carte des expositions dans laquelle la capitale française n'occuperait plus nécessairement la place centrale. Ainsi parmi les exposants étrangers de l'Exposition universelle de 1900, très peu avaient participé aux Salons parisiens. Comme le constate Robert de la Sizeranne en 1899, le temps de la «consécration parisienne » au Salon appartenait désormais au passé :

Dire que les artistes du monde entier doivent recevoir la consécration parisienne pour être acceptés chez eux est un aphorisme qui n'a pour lui que sa banalité. Il est tout à fait faux. Longue serait la liste des artistes Anglais, Allemands et même Italiens qui n'eurent aucun besoin de la consécration de nos Salons pour vendre leurs toiles aux plus riches collections d'Europe (La Sizeranne, 1899). 


\section{Conclusion}

Du point de vue de la participation des peintres étrangers, l'histoire du Salon dans la seconde moitié $d u x x^{e}$ siècle fait ressortir un phénomène d'internationalisation intimement lié à l'ère des expositions universelles. La politique d'internationalisation des expositions d'État mise en œuvre par l'administration du Second Empire a beaucoup contribué à l'affirmation de Paris comme centre artistique majeur, jusque bien après la chute du régime.

La complémentarité du Salon et des expositions universelles à Paris établit à partir de 1855 un véritable système de promotion des carrières artistiques qui fit émerger une élite internationale dont on observe, à partir des années 1880, la fin de l'unité.

La fin de cette unité, ou la crise du système du Salon, résulte de plusieurs phénomènes. L'évolution des structures d'exposition, d'une part: il apparaît avec la fin du Salon unique qu'une seule structure, aussi large soit-elle, ne pouvait plus assumer l'exposition et la promotion de la production artistique contemporaine dans son ensemble et sa diversité. Le succès relativement éphémère du Salon de la Société nationale des beaux-arts montre d'autre part l'épuisement d'une esthétique internationale propre à l'art du Salon. Et cette évolution du goût accompagne celle du statut de l'artiste étranger, qui demanda à être clarifié à partir des années 1880.

Paris demeura après 1900 un centre artistique extrêmement dynamique, continuant d'attirer en nombre les artistes étrangers, mais dans un contexte différent. La stigmatisation de l'étranger allait en effet s'y accentuer à l'approche de la Première Guerre mondiale. Tandis que le morcellement du paysage artistique de la capitale s'accentuait également, avec entre autres l'importance acquise par le Salon des Indépendants, créé dès 1884 ; l'apparition du Salon d'Automne en 1903, qui se distingua notamment par l'organisation d'expositions dédiées à l'art étranger; et enfin le développement d'un marché de l'art de plus en plus puissant et autonome.

Dans un contexte international, la crise du système du Salon interroge ainsi l'évolution de l'autonomie de l'artiste vis-à-vis des institutions officielles, mais aussi celle de ses rapports avec des composantes nationales inhérentes à l'art contemporain qui, loin de disparaitre, s'affirmeront sous des formes nouvelles au $\mathrm{xx}^{\mathrm{e}}$ siècle.

\section{BIBLIOGRAPHIE}

ALBERT Maurice (1890), « Le Salon de 1890 aux Champs-Élysées (1er article) », Gazette des BeauxArts (3), $1^{\text {er }}$ juin $1890,466$.

BOUSSAHBA-BRAVARD Myriam \& ROGERS Rebecca (2018), Women in international and universal exhibitions 1876-1937, Londres ; New York : Routeledge.

BRUNET François \& TALLEY Jessica (2017), « Exhibiting the West at the Paris Exposition of 1867: Towards a New American Aesthetic Identity? », Transatlantica (2). 
CAZES Laurent \& PENET Lyne (2017), « Beaux-arts et relations internationales sous le Second Empire : Théophile Silvestre et le projet d'une exposition d'art anglais au Salon de 1859 », Sociétés et représentations, 1(43), 251-272.

ChenNevières Philippe de (1853), Lettre autographe signée, 3 juin 1853, Paris : Archives des musées nationaux, X Salon 1853.

FINK Lois Marie (1990), American Art at the Nineteenth-Century Paris Salons, Cambridge : Cambridge University Press.

Gefrroy Gustave (1893), «Au Champ-de-Mars. Articles de Paris », La Vie artistique. Deuxième série, Paris : Dentu, 353-354.

JOYEUX-PRUNEL Béatrice (2016), Les avant-gardes artistiques 1818-1918. Une histoire transnationale, Paris : Gallimard.

KARP LUGo Laura (2014), Au-delà des Pyrénées : les artistes catalans à Paris au tournant du XXe siècle (thèse de doctorat), Université Paris 1 Panthéon-Sorbonne.

KEARNS James \& VAISSE Pierre (2010), Ce Salon à quoi tout se ramène. Le Salon de peinture et de sculpture, 1791-1890, Berne : Peter Lang.

KeARnS James \& Mill Alister (2015), The Paris Fine Art Salon / Le Salon, 1791-1881, Oxford ; Berne : Peter Lang.

KNels Eva (2019), Der Salon und die Pariser Kunstszene unter Napoleon I: Kunstpolitik, künstlerische Strategien, internationale Resonanzen, Hildesheim : Georg Olms.

LAGRANGE Marion (2010), Les peintres italiens en quête d'identité. Paris 1855-1909, Paris : cths / INHA.

LA SizerAnNe Robert de (1899), « Les Paysans aux Salons de 1899 », Revue des deux mondes, 153 (maijuin 1899), 407-434.

LOBSTEIN Dominique (2000), «Le Salon de la Société Nationale des Beaux-Arts, une création entre sécession et tradition ", G. Dugnat (dir.), Les Catalogues des Salons de la Société Nationale des BeauxArts, I (1890-1895), Paris : L'Échelle de Jacob, XI-XXIII.

MAINARDI Patricia (1987), Art and politics of the Second Empire, the universal expositions of 1855 and 1867, New Haven : Yale University press.

MAINARDI Patricia (1993), The end of the Salon: art and the state in the early Third Republic, Cambridge : Cambridge University Press.

MAINGON Claire (2009), Le Salon et ses artistes. Une histoire des expositions du Roi Soleil aux Artistes Français, Paris : Hermann.

NERLICH France (2010), Réception de la peinture française en Allemagne de 1815 à 1870, Paris : Éd. de la Maison des sciences de l'homme.

NoIRIEL Gérard (1999), « République et exclusion en France à la fin du XIXe siècle ", A. Gueslin \& D. Kalifa (dir.), Les exclus en Europe, 1830-1930, Paris : Les Éditions de l'Atelier / Éditions ouvrières, 265-273.

SfEIR-SeMLER Andrée (1992), Die Maler am Pariser Salon, 1791-1880, Frankfurt-am-Main ; New York : Campus Verl. ; Paris : Ed. de la Maison des sciences de l'homme.

TOLÈDE Olivia (2008), Une Sécession française : la Société nationale des beaux-arts (thèse de doctorat), Université de Paris-X, Nanterre ; Université de Genève, Genève.

VAISSE Pierre (1995), La Troisième République et les peintres, Paris : Flammarion. 
VAISSE Pierre (2010), « Réflexion sur la fin du Salon officiel », J. Kearns \& P. Vaisse (dir.), Ce Salon à quoi tout se ramène. Le Salon de peinture et de sculpture, 1791-1890, Berne : Peter Lang, 117-138.

VÉRAX Louis (1886), De l'Envahissement de l'École des Beaux-Arts par les Étrangers. Réclamations des élèves français. Temps perdu faute de places. Attribution des prix de fondations françaises. Avantages donnés aux étrangers par la nouvelle loi militaire, Paris : Libraire des imprimeries réunies.

VIRMAîTRE Charles (1890), Paris-Médaillé, 1890, Paris : L. Genonceaux.

VOTTERo Michaël (2012), La peinture de genre en France, après 1850, Rennes : Presses universitaires de Rennes.

WHITE Harisson C. \& WHITE Cynthia A. (1991 [1965]), La carrière des peintres au XIXe siècle, du système académique au marché des impressionnistes (A. Jaccottet, trad.), Paris : Flammarion.

ZoLA Émile (1970 [1880]), « Le Naturalisme au Salon », Le Voltaire, 18-22 juin 1880, Mon Salon, Manet, Écrits sur l'art, Paris : Garnier-Flammarion, 325-352.

\section{ANNEXES}

\section{Graphique 1}

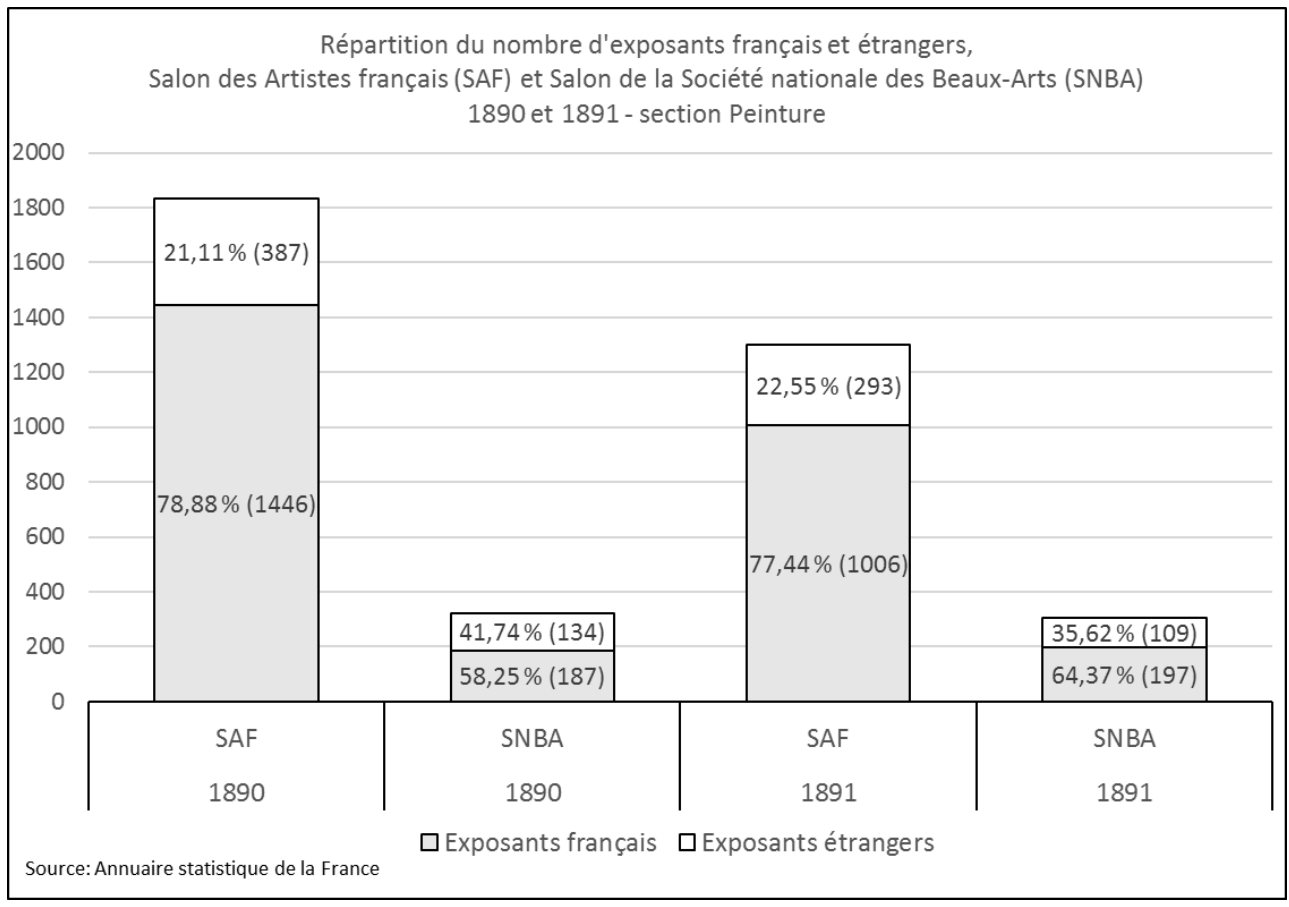




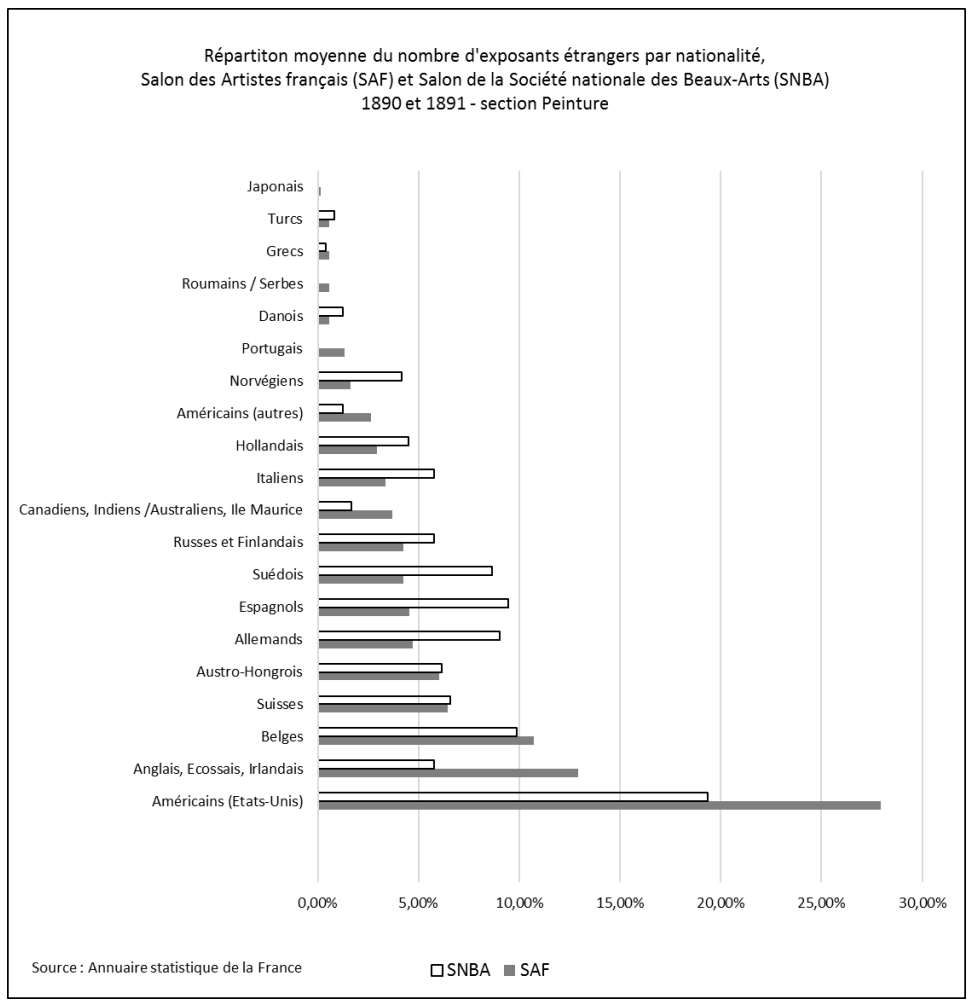

\section{NOTES}

1. En ce qui concerne la seconde moitié du XIX ${ }^{e}$ siècle, l'ouvrage pionnier d'Harrison et Cynthia White (1991 [1965]), fut suivi par l'ouvrage de Pierre Vaisse sur la Troisième république et les peintres (1995), qui reste la référence majeure. Pour des synthèses récentes sur le Salon parisien, voir Kearns \& Vaisse (2010), et Kearns \& Mill (2015).

2. Les réseaux constitués par les académies des beaux-arts européennes et extra européennes permirent de nombreux échanges transnationaux; voir notamment Nerlich (2010) pour le cas franco-allemand sur la période 1815-870.

3. Le critère encore flou de la nationalité, surtout avant les années 1880 ne permet pas d'établir de statistiques précises de la participation des artistes étrangers au Salon parisien. Cependant le dépouillement systématique des livrets de Salon, en prenant pour critère le lieu de naissance indiqué par les exposants, permet d'évaluer, dans la section peinture, une participation étrangère de $15 \%$ à $20 \%$ en moyenne sous le Second Empire ; cette moyenne descend à dix pour cent au Salon de 1872, puis remonte à $20 \%$ et reste stable à partir du milieu des années 1870 . Pour les statistiques de la participation étrangère aux Salons de 1890 et 1891 établies par l'Annuaire statistique de la France, voir graph. 1 et 2.

4. On ne dispose pas de chiffres précis sur la participation étrangère aux Salons de la première moitié $\mathrm{du} \mathrm{XIX}{ }^{\mathrm{e}}$ siècle. Dans son étude statistique sur les Salons Andrée Sfeir-Semler évoque environ $6 \%$ de peintres étrangers pour les Salons de 1845 et 1847 (Sfeir-Semler, 1992 : 259-262). Pour une étude récente sur les Salons de la période du Premier Empire, y compris dans une perspective transnationale, voir aussi Knels (2019).

5. Sous l'Ancien Régime, les livrets d'exposition de l'Académie royale indiquaient la qualité des artistes exposants (académicien, conseiller, etc.) ainsi que leurs éventuelles décorations. Après 
1791, seuls les adresses des exposants furent indiquées au livret, avec cependant parfois, durant la première décennie du XIX ${ }^{\mathrm{e}}$ siècle, l'indication du lieu de naissance et du maître.

6. Sur l'organisation de l'Exposition universelle de 1855, voir Mainardi (1987).

7. Il y eut deux exceptions, aux Salons de 1859 et de 1880, sur lequel je reviens plus loin. Sur la section étrangère du Salon de 1859, je me permets de renvoyer à notre article corédigé avec Lyne Penet (Cazes \& Penet, 2017).

8. Alma-Tadema avait participé aux Salons de 1864 (médaille) et 1865 ; Israëls aux salons de 1857, 1861 (mention honorable), 1863 et 1866 ; O. Achenbach aux Salons de 1859 (médaille de $3^{\mathrm{e}}$ classe), 1861 (médaille de $2^{\mathrm{e}}$ classe), 1863 (rappel de médaille de $2^{\mathrm{e}}$ classe et Croix de chevalier de l'ordre de la Légion d'honneur), 1864, 1865 et 1866 ; Knaus aux Salons de 1853 (médaille de $2^{\mathrm{e}}$ classe), 1857 (rappel de médaille de $1^{\text {re }}$ classe [Exposition universelle de 1855]), 1859 (rappel de médaille de $1^{\text {re }}$ classe [Exposition universelle de 1855] et Croix de chevalier de l'ordre de la Légion d'honneur) et 1863 ; Anker aux Salon de 1853, 1861, 1863 (mention honorable), 1864, 1865, 1866 (médaille); Palizzi aux Salons de 1853, 1857, 1859 (Croix de chevalier de l'ordre de la Légion d'honneur), 1861, 1863, 1864, 1865 et 1866 ; Pasini aux Salons de 1857, 1859 (médaille de $3^{\mathrm{e}}$ classe), 1861, 1863 (médaille de $2^{\mathrm{e}}$ classe), 1864 (médaille), 1865 et 1866.

9. À partir de 1867, la liste sépare les artistes français des artistes étrangers.

10. S'il y eut des exceptions, le principe voulait que les différentes classes de médailles soient progressivement décernées selon leur ordre hiérarchique, que venait couronner les décorations de la Légion d'honneur. Il n'était pas possible de "dégrader " un artiste en lui attribuant une récompense de valeur inférieure à celle(s) qu'il avait déjà obtenue(s).

11. Sur la création de la Société nationale des beaux-arts, voir Tolède (2008).

12. Voir les propos de Meissonier cités dans Virmaître (1890: 50-51).

\section{RÉSUMÉS}

À travers le prisme de la participation des peintres étrangers, cet article propose d'évoquer comment le Salon, associé aux expositions universelles françaises à partir de 1855, constitua un véritable système de promotion des carrières artistiques à l'échelle internationale. Ce système établit une élite artistique cosmopolite qui domina les grandes expositions de la seconde moitié du XIXe siècle, mais montra ses limites à partir des années 1880. Le désengagement de l'État de l'organisation du Salon entraîna un délitement de cette élite artistique et sa dispersion entre le Salon traditionnel et celui de la Société nationale des beaux-arts, créé en 1889. Tandis que le régime républicain amena à clarifier le statut de l'exposant étranger dans la sphère artistique parisienne, l'art cosmopolite du Salon qui brilla intensément durant les premiers Salons de la Société nationale des beaux-arts se trouva bientôt délaissé par les artistes et la critique. Le système du Salon laissa place à la fin du XIX ${ }^{e}$ siècle à une nouvelle carte des expositions qui se caractérise par sa diversité et une autonomie croissante.

Through the prism of the participation of foreign painters, this article proposes to evoke how the Salon, associated with the French Universal Exhibitions from 1855 onwards, constituted a real system for the promotion of artistic careers on an international scale. This system established a cosmopolitan artistic elite that dominated the major exhibitions of the second half of the 19th century, but showed its limits from the 1880s onwards. The disengagement of the State from the organization of the Salon led to the disintegration of this artistic elite and its dispersion between 
the traditional Salon and that of the Société nationale des beaux-arts, created in 1889. While the republican regime led to a clarification of the status of foreign exhibitors in the Parisian artistic sphere, the cosmopolitan art of the Salon, which shone intensely during the first Salons of the Sociéte nationale des Beaux-Arts, soon found itself abandoned by artists and critics. At the end of the 19th century, the Salon system gave way to a new geographic distribution of exhibitions characterized by its diversity and increasing autonomy.

\section{INDEX}

Mots-clés : salons et expositions artistiques, expositions universelles, Paris (France) xixe siècle, peintres étrangers, société des artistes français, société nationale des beaux-arts

Keywords : salons (exhibition), world fairs, Paris (France) 19th century, foreign artists, société des artistes français, société nationale des beaux-arts

\section{AUTEUR}

\section{LAURENT CAZES}

Docteur en histoire de l'art, Université Paris 1Panthéon-Sorbonne - HiCSA

lcazes@orange.fr 